\title{
Quantum error correction may delay, but also cause, entanglement sudden death
}

\author{
Isabel Sainz and Gunnar Björk \\ School of Information and Communication Technology, \\ Royal Institute of Technology (KTH), \\ Electrum 229, SE-164 40 Kista, Sweden
}

(Dated: October 26, 2018)

\begin{abstract}
Dissipation may cause two initially entangled qubits to evolve into a separable state in a finite time. This behavior is called entanglement sudden death (ESD). We study to what extent quantum error correction can combat ESD. We find that in some cases quantum error correction can delay entanglement sudden death but in other cases quantum error correction may cause ESD for states that otherwise do not suffer from it. Our analysis also shows that fidelity may not be the best measure to compare the efficiency of different error correction codes since the fidelity is not directly coupled to a state's remaining entanglement.
\end{abstract}

PACS numbers: 03.67.Mn, 03.65.Yz, 03.67.Pp 


\section{INTRODUCTION}

The view of entanglement has gone from being a consequence of quantum theory that "no reasonable definition of reality could be expected to permit" [1] to a physical resource on which quantum technologies are based [2]. For that reason it is important not only to understand entanglement dynamics, but it is desirable to be able to manipulate entanglement states in a practical way.

Entanglement dynamics has been studied in several contexts. When interacting with a reservoir, one would naively expect the entanglement between two systems to vanish asymptotically. However, inspired by the entanglement breaking quantum channel theorems [3], Diósi showed that if two systems are initially entangled, and they are subjected independently (or only one of them) to a depolarizing channel, the systems will become disentangled in a finite time [4]. This result was subsequently extended for continuous variables in [5]. Around the same time, Yu and Eberly [6] found that two initially entangled qubits placed in their own cavities can become disentangled via spontaneous emission at a rate larger than the decoherence rate. For certain initial states, the time of disentanglement is finite, a phenomenon termed "entanglement sudden death" (ESD) [7]. Lately, it has been found that ESD is present in different scenarios for certain classes of states, (see, for example, [8, 9, 10, 11] and the references therein). Moreover, this phenomenon was recently demonstrated in an experiment [12].

A worry, expressed in the literature, is that when an entangled state has suffered from entanglement sudden death due, e.g., to dissipation, and become disentangled, no (local) recovery operation can restore the state [11]. This means that the initial quantum resource carried by the state is irrevocably lost. ESD has hence been seen as a potential hurdle, or even impasse, for the development of, e.g., large scale quantum computing.

To combat the decoherence of quantum states, several schemes have been proposed during the last decade. When the considered subsystems are coupled to a collective environment, one can make use of decoherence-free subspaces [13]. Another technique, that does not necessarily require that the subsystems are coupled to the same environment, is the open-

loop control [14]. However, subsequently it was shown that the latter technique is the dynamical restoration equivalence of the decoherence-free subspaces [15, 16].

Another option to deal with noisy or dissipative channels is quantum error correction 
(QEC). In this scheme one can restore a "distorted" state by the introduction of redundancy by coding $[17,18,19,20,21,22,23,24,25,26]$. In general, QEC works in three stages: At the first stage, every physical qubit is encoded onto a logical qubit (code) composed of several physical qubits. The logical qubits then interact with a non-ideal (noisy) channel, that "distorts" the state. This distortion is unwanted, but will occur in most realistic channels. At the second stage, one performs a measurement to find the error syndrome, i.e., what kind of error the channel caused and in which physical qubit it occurred. In the third and final stage one restores the distorted logical state into the initial logical state by applying a recovery operation, conditioned on the information given by the error syndrome. Several well known error correcting codes exist [17, 18, 19, 20], and these enables one to correct a "general" error (e.g., represented by the Pauli operators) in a single qubit. (If two or more qubits are erroneous, more sophisticated codes must be used to restore the state.) Typically the loss of fidelity after correction is of the order of $\gamma^{2}$, where $\gamma$ is the probability of having one error in one of the physical qubits. This result should be compared with the fidelity without coding and error correction, where the loss of fidelity scales as $\gamma$.

In open quantum systems not all the possible errors can be represented by the discrete Pauli operators (that represent bitflips, phaseflips and both), e.g., amplitude damping. The codes like [17, 18, 19, 20] are not designed to correct open-system errors. Such errors do not fulfil the so-called QEC criteria [20, 21], and they can therefore not be exactly corrected. To address this problem, a channel-adapted quantum error correction scheme has been proposed in [23], where a code is presented that is able to correct a single general (discrete Pauli) error and is at the same time perfectly adapted to correct errors due to spontaneous emission. Concurrently, it was shown that small deviations from the QEC criteria will not change the fidelity qualitatively (in terms of the order of $\gamma$ ), and sufficient conditions for an approximate QEC were given [24]. Recently, numerical algorithms to generate channel-adapted recovery operations using well known codes such as [17, 18, 19, 20] were presented [25]. In [26] channel-adapted codes for an amplitude damping channel are developed, where the encoding is described in the stabilizer formalism. Different recovery operations are compared, specially optimal recovery and channel-adapted stabilizer recovery. The conclusion from [26] is that the improvement in fidelity by the optimal recovery is small compared to the cost of implementation.

In this paper, we will investigate to what extent ESD can be combatted using quantum 
coding adapted for dissipative channels. The hope is that the coding, which spreads the initial two-qubit entanglement over many qubits, may prevent ESD altogether. Somewhat surprisingly, the result is that while coding and error correction will indeed help for small amounts of dissipation, quantum coding actually makes matter worse when the dissipation is large. In particular, states that do not suffer from ESD when uncoded will, in coded form, display ESD.

\section{THE MODEL}

Entanglement sudden death is cased by dissipation. Consequently we will consider an amplitude damping channel, that for one qubit can be represented in terms of the Kraus operators :

$$
\hat{E}_{0}=\left(\begin{array}{cc}
1 & 0 \\
0 & \sqrt{1-\gamma}
\end{array}\right), \quad \hat{E}_{1}=\left(\begin{array}{cc}
0 & \sqrt{\gamma} \\
0 & 0
\end{array}\right)
$$

where $\gamma$ is the damping, $\hat{E}_{0}$ is the one-qubit no-jump operator that leaves the ground state $|0\rangle$ unchanged, but reduces the probability amplitude of the excited state $|1\rangle$ with the factor $1-\gamma$, and $\hat{E}_{1}$ represents the jump operator that transforms the state $|1\rangle$ into the state $|0\rangle$ with probability $\gamma$.

To investigate to what extent a two qubit state can be "protected" against amplitude damping and in particular to ESD, we will use the [4,1] code introduced in [24], where the notation $[4,1]$ denotes coding of each initial qubit onto a logical qubit consisting of four physical qubits. (We have also tested $[5,1]$ and $[6,2]$ codes with similar results.) The $[4,1]$ code encodes an arbitrary (single qubit) state $|\varphi\rangle=\cos \alpha|1\rangle+\sin \alpha|0\rangle$ into the logical state $|\varphi\rangle_{L}=\cos \alpha|1\rangle_{L}+\sin \alpha|0\rangle_{L}$, were the codewords $|0\rangle_{L}$ and $|1\rangle_{L}$, are given by the four qubit code [24, 26],

$$
\begin{aligned}
& |0\rangle_{L}=(|0000\rangle+|1111\rangle) / \sqrt{2} \\
& |1\rangle_{L}=(|0011\rangle+|1100\rangle) / \sqrt{2}
\end{aligned}
$$

where here, and below, we will use the notation $|0000\rangle \equiv|0\rangle \otimes|0\rangle \otimes|0\rangle \otimes|0\rangle$.

For simplicity we will suppose that all the physical qubits have the same damping probability $\gamma$. We will also assume that the amplitude damping channels are independent, which 
means that if the amplitude damping channel $\hat{E}$ acts on the one-qubit density matrix $\hat{\rho}$ as

$$
\hat{E}(\hat{\rho})=\hat{E}_{0} \hat{\rho} \hat{E}_{0}^{\dagger}+\hat{E}_{1} \hat{\rho} \hat{E}_{1}^{\dagger},
$$

the many-qubit amplitude damping channel will be described by the tensorial products of the corresponding Kraus operators, e.g., for two qubits, the channel will be described by operators of the form

$$
\hat{E}_{m n}=\hat{E}_{m} \otimes \hat{E}_{n}
$$

where $m, n=0,1$, and the first(second) operator acts on the first(second) qubit.

In our assumed channel we have a conditional evolution, because the ground state is not affected by the damping whereas the excited state is. Unfortunately, some well-know QEC protocols such as those in [17, 18, 19, 20] are not intended for this kind of channels. Nevertheless, using the same codewords one can find suitable syndrome measurements and recovery operators that do not fulfil the QEC conditions [21], but the "approximate" ones [24]. Hence, the $[4,1]$ code in (1) and (2) can only "approximately" correct no jump and one qubit jumps, in the sense that the distortion caused by the conditional evolution is of the second order in the damping parameter $\gamma$ [24].

As was pointed out in [26], the states reached from $|0\rangle_{L}$ and $|1\rangle_{L}$ through one-qubit jumping (i.e., removal of one excitation by the damping) are spanned by orthogonal subspaces. Every one of these subspaces are given by two vectors $\left\{\left|R_{i 0}\right\rangle,\left|R_{i 1}\right\rangle\right\}, i=0, \ldots, 4$, where $i=0$ denotes no jump, $i=1, \ldots, 4$ means a jump of (only) the first physical qubit and so on. In total 10 vectors spans the no-jump and one-qubit jump space. For no jump we have

$$
\left|R_{00}\right\rangle=|0\rangle_{L}, \quad\left|R_{01}\right\rangle=|1\rangle_{L},
$$

and for the one-qubit jump it is easy to obtain that

$$
\begin{array}{ll}
\left|R_{10}\right\rangle=|0111\rangle, & \left|R_{11}\right\rangle=|0100\rangle, \\
\left|R_{20}\right\rangle=|1011\rangle, & \left|R_{21}\right\rangle=|1000\rangle, \\
\left|R_{30}\right\rangle=|1101\rangle, & \left|R_{31}\right\rangle=|0001\rangle, \\
\left|R_{40}\right\rangle=|1110\rangle, \quad\left|R_{41}\right\rangle=|0010\rangle .
\end{array}
$$

In order to have a full four-qubit basis we can add the vectors:

$$
\left|R_{50}\right\rangle=(|0000\rangle-|1111\rangle) / \sqrt{2},
$$




$$
\begin{aligned}
& \left|R_{51}\right\rangle=(|0011\rangle-|1100\rangle) / \sqrt{2} \\
& \left|R_{60}\right\rangle=(|1010\rangle+|0101\rangle) / \sqrt{2}, \\
& \left|R_{61}\right\rangle=(|1001\rangle+|0110\rangle) / \sqrt{2}, \\
& \left|R_{70}\right\rangle=(|1010\rangle-|0101\rangle) / \sqrt{2}, \\
& \left|R_{71}\right\rangle=(|1001\rangle-|0110\rangle) / \sqrt{2}
\end{aligned}
$$

It is not important how one chooses these additional basis elements since they do not correspond to any correctable error.

In this basis, let us introduce the following unitary transformation to make the syndrome measurement more transparent:

$$
\hat{U}=\sum_{k=0}^{7} \sum_{i=0}^{1}|i \operatorname{Bin}(k)\rangle\left\langle R_{k i}\right|,
$$

where $\operatorname{Bin}(k)$ is the three-bit, binary representation of $k=0 \ldots, 7$. In an experimental realization, this transformation can be incorporated in the syndrome measurement.

With the transformation given above one can approximately detect no jump in any physical qubit, and one-qubit jumps by measuring the three rightmost qubits in the computational basis. If the result is 000 there has been no jump, while the results 001, 010, 011, and 100 indicate that there have been a jump in qubits 1-4, respectively. Actually, e.g., the result 010 can also indicate jumps in all of qubits 2,3 , and 4 from the state $|0\rangle_{L}$. However, this code is not designed to cope with multiple-qubit jumps, so it will act on this syndrome as if it was caused by a single qubit jump in qubit 2 .

Once the syndrome is measured we will apply the corresponding recovery operation (summarized in the table below): If we measure $\operatorname{Bin}(k)$ for $k=0, \ldots, 4$, we will preserve the possible superposition carried by the first qubit by implementing the transformation $\hat{R}_{k}=\sum_{i=0}^{1}|i\rangle_{L}\langle i \operatorname{Bin}(k)|$. If we happen to measure the syndromes $\operatorname{Bin}(k)$ for $k=5,6$, or 7, we know multiple (but not which) jumps have occurred. Hence we cannot correct the state but chose to project the system to the state $\hat{I}_{L} / 2$, where $\hat{I}_{L}=|0\rangle_{L}\left\langle\left. 0\right|_{L}+\mid 1\right\rangle_{L}\left\langle\left. 1\right|_{L}\right.$ is the identity operator in the codeword space. After correction, the state is always in a four-dimensional codeword-space enabling one to compute (or measure) the fidelity to the initial state or the concurrence in the standard fashion. 
TABLE I: The syndromes and corresponding recovery transformations.

\begin{tabular}{|c|c|}
\hline Syndrome & Recovery transformation \\
\hline $\operatorname{Bin}(0)=000$ & $\hat{R}_{0}=\sum_{i=0}^{1}|i\rangle_{L}\langle i \operatorname{Bin}(0)|$ \\
$\operatorname{Bin}(1)=001$ & $\hat{R}_{1}=\sum_{i=0}^{1}|i\rangle_{L}\langle i \operatorname{Bin}(1)|$ \\
$\operatorname{Bin}(2)=010$ & $\hat{R}_{2}=\sum_{i=0}^{1}|i\rangle_{L}\langle i \operatorname{Bin}(2)|$ \\
$\operatorname{Bin}(3)=011$ & $\hat{R}_{3}=\sum_{i=0}^{1}|i\rangle_{L}\langle i \operatorname{Bin}(3)|$ \\
$\operatorname{Bin}(4)=100$ & $\hat{R}_{4}=\sum_{i=0}^{1}|i\rangle_{L}\langle i \operatorname{Bin}(4)|$ \\
\cline { 2 - 2 } $\operatorname{Bin}(5)=101$ & Project onto \\
$\operatorname{Bin}(6)=110$ & $\hat{I}_{L} / 2$ \\
$\operatorname{Bin}(7)=111$ & \\
\hline
\end{tabular}

\section{DOES QUANTUM ERROR CORRECTION PREVENT ENTANGLEMENT SUDDEN DEATH?}

As pointed out in the introduction, it has been shown that ESD is present in different scenarios for some bipartite states [4, 5, 6, 7, 8, 9, 10, 11, 12]. In particularl, the so-called "X-states" [8] have been widely studied [9]. They are called X-states because their composite density matrix has non-zero elements only in its diagonal and antidiagonal, if expressed in the basis $\{|00\rangle,|01\rangle,|10\rangle,|11\rangle\}$. They also have the property that the corresponding density matrix preserves the $\mathrm{X}$ form when evolving under the action of certain system dynamics, and in particular under dissipation. The class contain the Bell-states and the Werner-states and therefore merits studying. The class also encompasses both states that succumb to ESD and states that does not. To limit our study somewhat, we will work with the Bell-like X-states of the form

$$
\begin{aligned}
& \left|\phi_{0}\right\rangle=\cos \alpha|11\rangle+e^{i \beta} \sin \alpha|00\rangle \\
& \left|\psi_{0}\right\rangle=\cos \alpha|10\rangle+e^{i \beta} \sin \alpha|01\rangle .
\end{aligned}
$$

For comparison, we shall also study the separable, non-X-state

$$
\left|\varphi_{0}\right\rangle=\cos \alpha|11\rangle+e^{i \beta} \sin \alpha|10\rangle
$$


The state $\left|\phi_{0}\right\rangle$ will suffer from ESD when $|\tan \alpha|<1$. This was experimentally demonstrated in [12]. Meanwhile, $\left|\psi_{0}\right\rangle$ does not, and $\left|\varphi_{0}\right\rangle$ certainly does not, as is separable.

We shall now try to protect the information and entanglement carried by the states (7)(9) from the action of independent amplitude damping channels, and particularly from ESD (if present) by encoding each qubit using the codewords (11) and (2), so that we obtain the logical states

$$
\begin{aligned}
& |\phi\rangle=\cos \alpha|11\rangle_{L}+e^{i \beta} \sin \alpha|00\rangle_{L}, \\
& |\psi\rangle=\cos \alpha|10\rangle_{L}+e^{i \beta} \sin \alpha|01\rangle_{L}, \\
& |\varphi\rangle=\cos \alpha|11\rangle_{L}+e^{i \beta} \sin \alpha|10\rangle_{L} .
\end{aligned}
$$

To characterize to what extent the states are "protected" by coding and error correction we will use the fidelity to the initial state and the concurrence.

After damping and error correction the fidelities between the states (10)-(12) and the corresponding undamped states, are given (to the second order in $\gamma$ ) by:

$$
\begin{aligned}
\mathcal{F}_{\phi}= & 1-\frac{\gamma^{2}}{2}(7-3 \cos 2 \alpha-\cos 4 \alpha)+O\left(\gamma^{3}\right) \\
\mathcal{F}_{\psi}= & 1-\frac{\gamma^{2}}{2}(7-\cos 4 \alpha)+O\left(\gamma^{3}\right) \\
\mathcal{F}_{\varphi}= & 1-\frac{\gamma^{2}}{8}(17-6 \cos 2 \alpha+\cos 4 \alpha \\
& \left.-2 \cos 2 \beta \sin ^{2} 2 \alpha\right)+O\left(\gamma^{3}\right)
\end{aligned}
$$

Meanwhile, the fidelities between the initial and damped corresponding uncoded states are:

$$
\begin{aligned}
\mathcal{F}_{\phi_{0}}= & 1-2 \gamma \cos ^{2} \alpha+\gamma^{2} \cos ^{2} \alpha \\
\mathcal{F}_{\psi_{0}}= & 1-\gamma \\
\mathcal{F}_{\varphi_{0}}= & 1-\frac{\gamma}{8}(11+4 \cos 2 \alpha+\cos 4 \alpha) \\
& +\frac{\gamma^{2}}{8} \cos ^{2} \alpha(3+5 \cos 2 \alpha)+O\left(\gamma^{3}\right) .
\end{aligned}
$$

As expected, the loss of fidelity obtained is of the order of $\gamma^{2}$ for the error corrected states while it is linear for the uncoded states. The exception is $\mathcal{F}_{\phi_{0}}$ that approaches unity when $\alpha \rightarrow \pi / 2$. This is to be expected since, in this limit, the state is the trivial, nonevolving, ground state. 

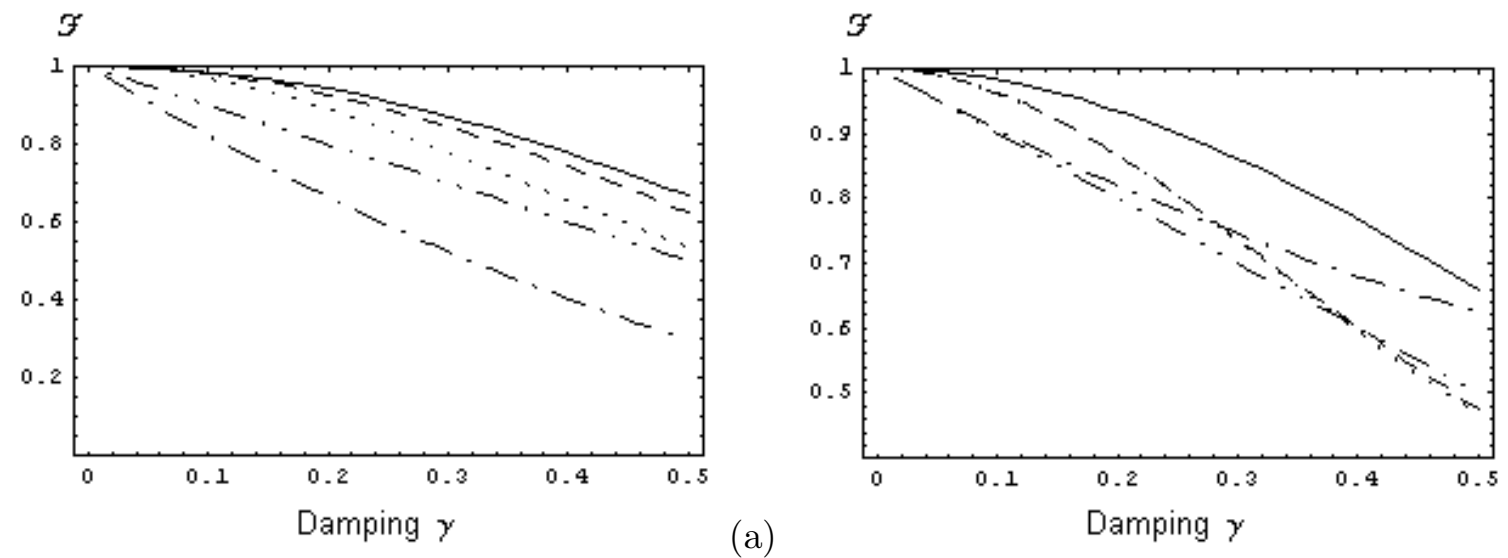

FIG. 1: Plot of the fidelity after recovering for the states $|\phi\rangle$ (dashed), $|\psi\rangle$ (dotted), and $|\varphi\rangle$ (continuous) together with the fidelity for the uncoded states $\left|\phi_{0}\right\rangle$ (dash-dotted) and $\left|\psi_{0}\right\rangle$ (dashdouble dotted). The parameters are $\beta=0$, (a) $\alpha=\pi / 12$ and (b) $\alpha=\pi / 4$. (In (b) the curves for $|\phi\rangle$ and $|\psi\rangle$ almost coincide.)

The expressions for the concurrence $\mathcal{C}$ of the states are analytically "messy", and therefore we have chosen to simply plot the exact, numerically results. The plotted fidelities are also based on the exact expressions and not on the series expansions (13)-(18). Since the dependence of on $\beta$ is weak, we have, rather arbitrarily, set $\beta=0$ when making the plots. In Fig. 11 we can compare the fidelities (13)-(17). In general, the fidelity as a function of the damping will be larger for the unentangled state $|\varphi\rangle$ than for the states $|\phi\rangle$ and $|\psi\rangle$. The fidelity for the state $|\phi\rangle$ is larger than the one for $|\psi\rangle$ for $\alpha<\pi / 4$, for $\alpha<\pi / 4$ the opposite happen, being roughly the same when $\alpha=\pi / 4$.

In Fig. 2 we plot the fidelities and concurrences for the coded state $|\phi\rangle$ after error correction, and the corresponding uncoded state $\left|\phi_{0}\right\rangle$ as a function of the damping. In Figure 2 (a) it is seen that the quantum error correction makes the state almost twice as tolerant to damping before the entanglement vanishes. However, this is not a general truth, and this can be seen in 2 (b) where the coded state suffer from ESD while the uncoded state does not (because for the uncoded state $\mathcal{C}=0$ only as $\gamma=1$ ). What is also clear is that for small values of $\gamma$, quantum error correction increases both the fidelity and the entanglement. 

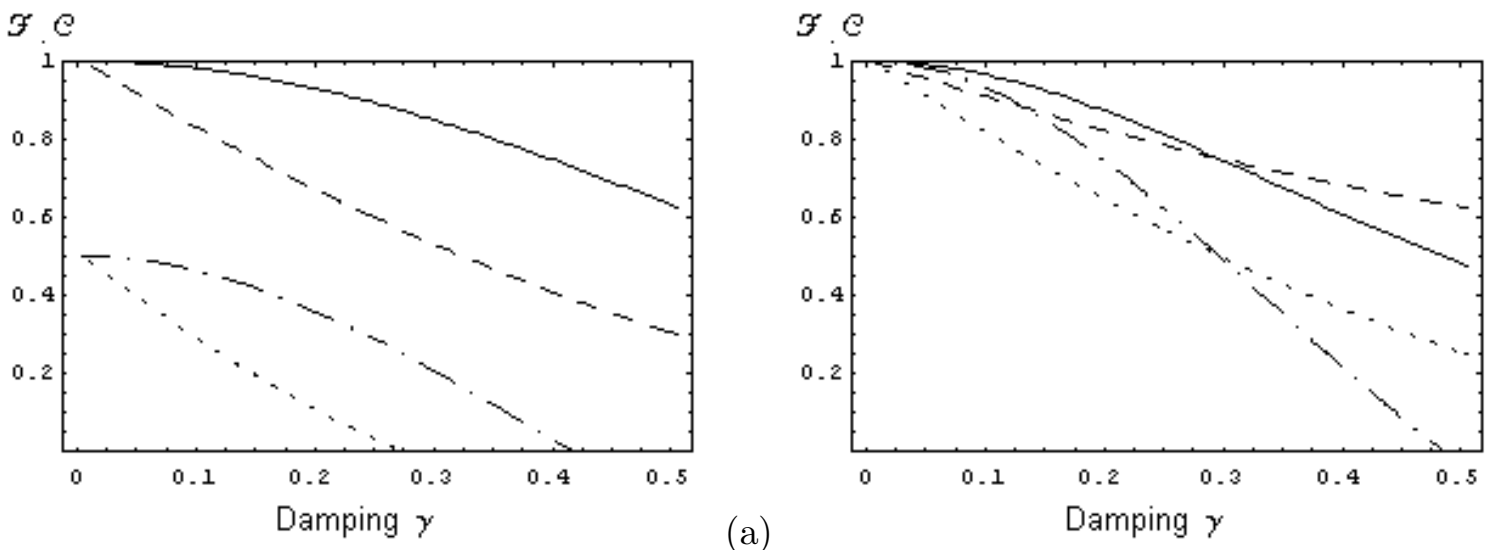

FIG. 2: Plot of the fidelity (continuous) and the concurrence (dash-dotted) for the state $|\phi\rangle$ after amplitude damping and recovery, together with the fidelity (dashed) and the concurrence (dotted) for the uncoded state $\left|\phi_{0}\right\rangle$. The parameters are $\beta=0$, (a) $\alpha=\pi / 12$ and (b) $\alpha=\pi / 4$.
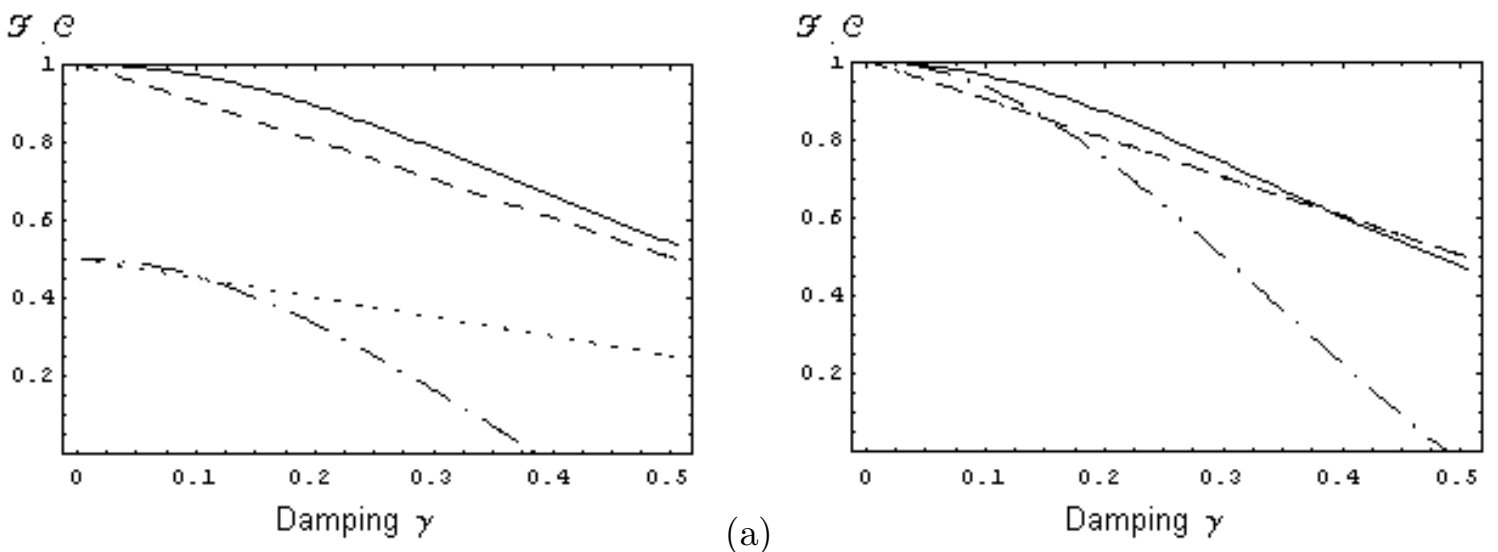

FIG. 3: Plot of the fidelity (continuous) and the concurrence (dash-dotted) for the state $|\psi\rangle$ after amplitude damping and recovery, together with the fidelity (dashed) and the concurrence (dotted) for the uncoded state $\left|\psi_{0}\right\rangle$. The parameters are $\beta=0$, (a) $\alpha=\pi / 12$ and (b) $\alpha=\pi / 4$.

In Fig. 3 we plot the fidelities and concurrences for the logical state $|\psi\rangle$ and for the corresponding uncoded state $\left|\psi_{0}\right\rangle$. When $\alpha=\pi / 4$, the concurrence and the fidelity for the uncoded state $\left|\psi_{0}\right\rangle$ have the same value, $1-\gamma$, and the curves in Figure [3(b), are not distinguishable. Here again, we see that for large values of $\gamma$ the coded state loses its entanglement in a finite time, while the uncoded state only looses its entanglement asymptotically so that $\mathcal{C}=0$ only for $\gamma=1$. However, for small values of $\gamma$ both the fidelity and the concurrence is increased by the use of quantum error correction, as expected. 


\section{CONCLUSIONS}

With a few illustrative examples we have studied the possibility of "protecting" two-qubit, pure, Bell-like states from the action of amplitude damping. As we have seen, quantum error correction succeeds in protecting the state to first order in the dissipation parameter, but when the dissipation increases beyond a certain point, quantum error correction often makes matters worse and not better. While longer and more complex codes may protect the states to higher orders in the dissipation parameter, we conjecture that the change is quantitative rather than qualitative compared to simpler codes. In particular, we find that quantum error correction will not protect a state from entanglement sudden death. On the contrary, states that do not succumb to entanglement sudden death may do so after they are coded.

Our examples also show what is perhaps obvious, but not much discussed, namely that fidelity is insufficient to quantify quantum processes and protocols. In all cases, the fidelity decreases asymptotically with increasing imperfections. However, the resource that is often of primary interest in quantum information, the entanglement, does not decrease asymptotically. After suffering a finite amount of dissipation the entanglement is gone, while the fidelity may still seem comfortably high. The fidelity may thus be deceptive as a figure of merit.

While we have only presented results for the $[4,1]$-code (applied to the two qubits separately), we have also made similar analyses for the [5,1]-code given in [19, 20] and the $[6,2]$-code in [26] (that encodes both qubits together). These codes will give marginally better results in the fidelity and concurrence, with similar syndrome measurement and recovery operations, but the qualitative behavior, discussed above, remains. However, the [5, 1]-code is a more complex code and will be more difficult to implement in practice. The [6,2] code, in turn, is a nonlocal code and employs non-local recovery operations. Hence, its implementation requires creation of entanglement over the same physical distances as the entangled qubits. This is a substantial drawback of the code.

Our final conclusion is not surprising. In order to combat loss of entanglement due to dissipation one must employ quantum error correction. It is important, however, to apply

the recovery operation while the dissipation is still minor, say, $\gamma \leq 0.1$. For values of $\gamma \geq 0.5$ the entanglement may already have vanished, and will typically have done so if coding for quantum error correction has been used. 


\section{Acknowledgements}

This work was supported by the Swedish Foundation for International Cooperation in Research and Higher Education (STINT), the Swedish Research Council (VR), and the Swedish Foundation for Strategic Research (SSF).

[1] A. Einstein, B. Podolsky, and N. Rosen, Phys. Rev. 47, 777 (1935).

[2] M. Nielsen and I. Chuang, Quantum Computation and Quantum Information (Cambridge University Press, Cambridge, 2000).

[3] A. S. Holevo, Russian Math. Surveys 53, 1295 (1998); P. W. Shor, J. Math. Phys. 43, 4334 (2002); M. Horodecki, P. W. Shor, and M. B. Ruskai Rev. Math. Phys. 15, 629 (2003).

[4] L. Diósi, in Proceedings of the Conference on Decoherence, Information, Complexity and Entropy (DICE), edited by H. T. Elze (Piombino, Italy 2002).

[5] P. J. Dodd and J. J. Halliwell, Phys. Rev. A 69, 052105 (2004); P. J. Dodd ibid. 69, 052106 (2004).

[6] T. Yu and J. H. Eberly, Phys. Rev. Lett. 93, 140404 (2004).

[7] T. Yu and J. H. Eberly, Opt. Commun. 264, 393 (2006).

[8] T. Yu and J. H. Eberly, Quantum Inf. Comp. 7, 459 (2007).

[9] L. Jakóbczyk and A. Jamróz, Phys. Lett. A 333, 35 (2004); M. F. Santos, P. Milman, L. Davidovich, and N. Zagury, Phys. Rev. A 73, 040305(R) (2006); T. Yu and J. H. Eberly, Phys. Rev. Lett. 97, 140403 (2006).

[10] M. Lucamarini, S. Paganelli, and S. Mancini, Phys. Rev. A 69, 062308 (2004).

[11] J. H. Eberly and T. Yu, Science 316, 555 (2007).

[12] M. P. Almeida, F. de Melo, M. Hor-Meyll, A. Salles, S. P. Walborn, P. H. S. Riberio, and L. Davidovich, Science 316, 579 (2007).

[13] G. M. Palma, K.-A. Suominen, and A. K. Ekert, Proc. R. Soc. London A 452, 567 (1996); I. L. Chuang and Y. Yamamoto, Phys. Rev. Lett. 76, 4281 (1996); P. Zanardi and M. Rasetti, Phys. Rev. Lett. 79, 3306 (1997); L.-M. Duan and G.-C. Guo, Phys. Rev. Lett. 79, 1953 (1997).

[14] L. Viola and S. Lloyd, Phys. Rev. A 58, 2733 (1998). 
[15] P. Zanardi, Phys. Lett. A 258, 77 (1999); L. Viola, E. Knill, and S. Lloyd, Phys. Rev. Lett. 82, 2417 (1999); L. Viola, E. Knill, and S. Lloyd, Phys. Rev. Lett. 85, 3520 (2000); L. A. Wu and D. A. Lidar, Phys. Rev. Lett. 88, 207902 (2002); P. Facchi, D. A. Lidar, and S. Pascazio, Phys. Rev. A 69, 032314 (2004); E. Brion et al., Phys. Rev. A 71, 052311 (2005).

[16] E. Knill, R. Laflamme, and L. Viola, Phys. Rev. Lett. 84, 2525 (2000).

[17] P. W. Shor, Phys. Rev. A 52, R2493 (1995).

[18] A. M. Steane, Phys. Rev. Lett. 77, 793 (1996).

[19] R. Laflamme, C. Miquel, J. P. Paz., and W. H. Zurek., Phys. Rev. Lett. 77, 198 (1996).

[20] C. H. Bennett, D. P. DiVincenzo, J. A. Smolin, and W. K. Wootters, Phys. Rev. A 54, 3824 (1996).

[21] A. Ekert and C. Macchiavello, Phys. Rev. Lett. 77, 2585 (1996); E. Knill and R. Laflamme, Phys. Rev. A 55, 900 (1997).

[22] D. Gottesman, Phys. Rev. A 54, 1862 (1996); A. R. Calderbank, E. M. Rains, P. W. Shor, and N. J. A. Sloane, Phys. Rev. Lett. 78, 405 (1997).

[23] M. B. Plenio, V. Vedral, and P. L. Knight, Phys. Rev. A 55, 67 (1997).

[24] D. W. Leung, M. A. Nielsen, I. L Chuang, and Y. Yamamoto, Phys. Rev. A 56, 2567 (1997).

[25] A. S. Fletcher, P. W. Shor, and M. Z. Win, Phys. Rev. A 77, 012320 (2008).

[26] A. S. Fletcher, P. W. Shor, and M. Z. Win, preprint: quant-ph/0710.1052 (2007). 